\title{
Expert consensus document on the measurement of aortic stiffness in daily practice using carotid-femoral pulse wave velocity
}

\author{
Luc M. Van Bortel ${ }^{a}$, Stephane Laurent ${ }^{b}$, Pierre Boutouyrie $^{b}$, Phil Chowienczyk $^{c}$, J.K. Cruickshank ${ }^{d}$, \\ Tine De Backer ${ }^{a}$, Jan Filipovsky ${ }^{e}$, Sofie Huybrechts ${ }^{a}$, Francesco U.S. Mattace-Raso ${ }^{f}$, \\ Athanase D. Protogerou ${ }^{g}$, Giuseppe Schillaci ${ }^{\text {h }}$, Patrick Segersi ${ }^{i}$, Sebastian Vermeersch ${ }^{a}$, \\ Thomas Weber ${ }^{j}$, on behalf of the Artery Society, the European Society of Hypertension Working \\ Group on Vascular Structure and Function and the European Network for Noninvasive \\ Investigation of Large Arteries
}

Stiffness of elastic arteries like the aorta predicts cardiovascular risk. By directly reflecting arterial stiffness, having the best predictive value for cardiovascular outcome and the ease of its measurement, carotid-femoral pulse wave velocity is now considered the gold standard for arterial stiffness assessment in daily practice. Many different measurement procedures have been proposed. Therefore, standardization of its measurement is urgently needed, particularly regarding the distance measurement. This consensus document advises on the measurement procedures in general and provides arguments for the use of $80 \%$ of the direct carotid-femoral distance as the most accurate distance estimate. It also advises the use of $10 \mathrm{~m} / \mathrm{s}$ as new cut-off value for carotid-femoral pulse wave velocity.

Keywords: arterial stiffness, carotid-femoral pulse wave velocity, expert consensus, measurement procedures

Abbreviation: PWV, pulse wave velocity

$\mathrm{M}$ any studies have shown that the stiffness of elastic arteries like the aorta independently predicts cardiovascular risk [1-3]. The predictive value of aortic stiffness, measured as carotid-femoral pulse wave velocity (PWV), was largely superior in the Framingham Heart Study to brachial artery stiffness (carotid-radial PWV), augmentation index, central pulse pressure and pulse pressure amplification [4]. The same holds in patients with end-stage renal disease, with carotid-femoral PWV (cfPWV) also superior to brachial artery and femorotibial stiffness [5]. By directly reflecting arterial stiffness, having the best predictive value for cardiovascular events and the ease of its measurement, cfPWV is now considered the gold standard for arterial stiffness assessment [1]. The 2007 European Society of Hypertension-European Society of Cardiology (ESH-ESC) Guidelines for the Management of Hypertension consider a high cfPWV as target organ damage [6].

Carotid-femoral PWV is calculated by dividing traveled distance by transit time (PWV= distance/time). The large majority of devices quite accurately measure transit time as the time delay between the arrival of the pulse wave at the common carotid artery and the common femoral artery. This measurement can be done directly on the same pulse wave, or indirectly by subtracting the time delay between the $R_{\text {top }}$ of the electrocardiogram to the common carotid artery from the time delay between the $R_{\text {top }}$ of the electrocardiogram to the common femoral artery. The traveled distance should also be measured accurately since small differences may influence the absolute value of PWV $[1,7]$. However, there is no consensus on how this distance is ideally measured and many different distances have been proposed (Table 1). A standardization of the measurement technique is requested since each distance definition results in a different PWV value $[8,9]$ increasing confusion among users. Whereas the cut-off value of $12 \mathrm{~m} / \mathrm{s}$ in the European guidelines [6] only applies to the full (100\%) direct carotidfemoral (common carotid artery-common femoral artery) distance, the recently published reference values are based on $80 \%$ of the common carotid artery-common

Journal of Hypertension 2012, 30:445-448

aHeymans Institute of Pharmacology, Ghent University, Ghent, Belgium, ' Department of Pharmacology and INSERM U970, Hôpital Européen Georges Pompidou, ParisDescartes University, Paris, France, ${ }^{C}$ Cardiovascular Division, Department of Clinical Pharmacology, King's College London British Heart Foundation Centre, 'King's College London, Diabetes, Nutrition and Cardiovascular Divisions, St. Thomas' Hospital, London, UK, 'Second Department of Internal Medicine, Medical Faculty and University Hospital, Charles University, Pilsen, Czech Republic, ${ }^{\mathrm{f}}$ Department of Internal Medicine, Erasmus Medical Center, Rotterdam, The Netherlands, ${ }^{9}$ Hypertension Center, 1st Department of Propaedeutic and Internal Medicine, Laiko Hospital, National and Kapodistrian University of Athens, Athens, Greece, 'Unit of Internal Medicine, Angiology and Arteriosclerotic Disease, University of Perugia Medical School, Hospital 'S. Maria della Misericordia', Perugia, Italy, 'IBiTech-bioMMeda, Ghent University, Ghent, Belgium and ${ }^{\mathrm{j} C}$ ardiology Department, Klinikum WelsGrieskirchen, Wels, Austria

Correspondence to Professor Luc M. Van Bortel, MD, PhD, Clinical Pharmacology and Pharmacotherapy, Heymans Institute of Pharmacology, Ghent University, Drug Research Unit Ghent (D.R.U.G.), Ghent University Hospital De Pintelaan 185, 9000 Ghent, Belgium. Tel: +32 9332 5800; fax: +32 9332 8959; e-mail: luc.vanbortel @ugent.be

Received 11 November 2011 Accepted 24 November 2011

J Hypertens 30:445-448 (C) 2012 Wolters Kluwer Health | Lippincott Williams \& Wilkins.

DOI:10.1097/HJH.0b013e32834fa8b0 
TABLE 1. Comparison of MRI measured traveled distance (reference distance) and estimated distance (from [11])

\begin{tabular}{|c|c|c|c|}
\hline Reference distance mean (SD) & Tape measure distance mean (SD) & & $\begin{array}{l}\text { Tape measure - reference } \\
\text { distance mean (SD) }\end{array}$ \\
\hline$(A A-F A)-(A A-C A)$ & $(C A-F A)-(C A-S N)$ & $48.3(4.1)$ & $-2.4(3.8)^{*}$ \\
\hline \multirow[t]{10}{*}{$50.7(4.2)$} & $(C A-F A)-(C A-S S N)$ & $53.0(4.1)$ & $2.3(3.8)^{*}$ \\
\hline & $(S N-F A)-(C A-S N)$ & $35.9(4.3)$ & $-14.8(3.9)^{*}$ \\
\hline & $(S S N-F A)-(C A-S S N)$ & $45.5(4.5)$ & $-5.1(3.5)^{*}$ \\
\hline & $(S S N-U M B)+(U M B-F A)$ & $57.7(4.5)$ & $7.2(4.0)^{*}$ \\
\hline & $(C A-F A)$ & $63.6(4.4)$ & $13.0(4.2)^{*}$ \\
\hline & {$[(\mathrm{SSN}-\mathrm{UMB})+(\mathrm{UMB}-\mathrm{FA})]-(\mathrm{CA}-\mathrm{SN})$} & $42.3(4.7)$ & $-8.2(4.1)^{*}$ \\
\hline & {$[(\mathrm{SSN}-\mathrm{UMB})+(\mathrm{UMB}-\mathrm{FA})]-(\mathrm{CA}-\mathrm{SSN})$} & $47.0(4.7)$ & $-3.5(4.1)^{*}$ \\
\hline & $(C A-F A) \times 0.8$ & $50.9(3.5)$ & $0.3(3.8)$ \\
\hline & Body height/4 + C (7.28) & $50.2(2.3)$ & $-0.5(3.9)$ \\
\hline & Body height $\times 0.29$ & $49.8(2.6)$ & $-0.9(4.0)^{\dagger}$ \\
\hline
\end{tabular}

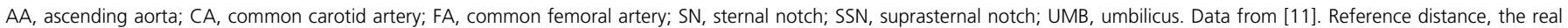
traveled aortic path length determined by MRI; tape measure distance, body surface distance obtained by tape measure.

${ }^{*} P<0.001$.

${ }^{\dagger} P<0.05$.

femoral artery distance [7]. In addition, although conversion formulas have been proposed to calculate subtracted distance (suprasternal notch to common femoral artery minus suprasternal notch to common carotid artery) from direct distance and vice versa [10], these formulas are approximations and introduce additional error.

Recently different proposed distances have been compared with the real traveled distance measured by magnetic resonance imaging (MRI). From the MRI images a centerline was constructed in the lumen of the arteries. Following these arterial centerlines, the real traveled distance was defined as the ascending aorta (from aortic valve) to right common femoral artery distance minus ascending aorta to right common carotid artery distance [11]. This study showed that the direct carotid-femoral distance largely overestimates the real traveled distance by $25.4 \%$, whereas the subtracted distances using the distances to common femoral artery and common carotid artery from suprasternal and sternal notch substantially underestimate the real traveled distance by 10.3 and $29.2 \%$, respectively. Of all currently used distances the $80 \%$ of the direct carotid-femoral distance (common carotid artery - common femoral artery $\times 0.8)$ appeared the most accurate, only slightly overestimating the real traveled distance by $0.4 \%$. Two other estimates performing relatively well were both based on body height (body height $/ 4+7.28$ proposed by Weber et al. [12] and body height $\times 0.29$ proposed by Filipovsky et al. [13]) underestimating the traveled distance by 1.0 and $1.8 \%$, respectively. The model obtained from a multiple linear regression analysis $\left(r^{2}=0.64\right)$ was: real traveled distance $=(0.286 \times$ common carotid artery - common femoral artery $)+(0.101 \times$ age $)+$ $(0.159 \times$ weight $)+16.165$, with common carotid artery common femoral artery in $\mathrm{cm}$, age in years and weight in $\mathrm{kg}$.

This best fit formula appears too complicated to be applied in daily practice, unless built in a dedicated device.

The same study also confirmed the data by Sugawara et al. [14], showing a distance which increases with age. In the population studied by Huybrechts et al. [11], the variation in distance between the (common carotid artery - common femoral artery $\times 0.8$ ) tape measure distance and the MRI measured real traveled distance was largely explained by increasing age (30\%) and to a much smaller extent by body mass index (BMI; 4\%). Therefore, it was tested in the same population whether a simple and for daily practice acceptable modification of the (common carotid artery - common femoral artery $\times 0.8)$ distance taking into account age would be more accurate. The tested formula adjusts the (common carotid artery-common femoral artery $\times 0.8)$ distance for age by adding $1 \mathrm{~mm} /$ year for an age above 50 and subtracting $1 \mathrm{~mm} /$ year for an age below 50, respectively (Table 2). Although on the population level (Table 2a) this age-adjusted formula performed better; this was not the case on an individual level in the age group between 50 and 76 years (Table 2b), which may be considered the more relevant group for targeting. More data in the very elderly may be needed. Thus, at this stage, we advise to use the simple unadjusted (common carotid artery - common femoral artery $\times 0.8$ ) distance as standard distance for daily practice, for three main reasons: the age adjustment did not perform better in the older age group; the adjusted formula is less easy to use in daily practice; and as in the study by Sugawara et al. [14] the age-dependent elongation of the aortic path length is limited.

This consensus panel is aware that the so-called 'real' traveled distance of cfPWV is not a simple unidirectional path length since at the time the pulse wave travels up the brachiocephalic and carotid artery, it also travels down the aortic arch. MRI measurements as outlined above are valid only after the assumption that these velocities are the same in both directions, and this may not be the case. In animals PWV in the carotid artery may be higher by $2-3 \mathrm{~m} / \mathrm{s}$ than in the aortic arch [15]. Rough calculations from studies showing both common carotid stiffness and cfPWV suggest that the difference in PWV between common carotid artery and aortic arch/descending aorta may be smaller in humans [16] and is probably affecting cfPWV less than $3 \%$. The present data also are based on measurements at the right common carotid artery and common femoral artery. It is not excluded that this may be slightly different for measurements at the left side.

The current cut-off value, proposed in the 2007 ESHESC Guidelines for the Management of Hypertension [6], is $12 \mathrm{~m} / \mathrm{s}$, above which there is an increased risk for cardiovascular events. This value was selected because it corresponded to a rough estimate of high cardiovascular risk 
TABLE 2. Error of distance estimation (CA $-F A) \times 0.8$ and the age-adjusted variant $[(C A-F A) \times 0.8+(0.1 \times($ age-50)] compared to MRI measured reference distance

(a) Mean difference from reference distance

Age $<50(N=52)$

\section{$49.0(3.2)$}

$2.05(3.3) *$

$0.44(3.3)$

$(\mathrm{CA}-\mathrm{FA}) \times 0.8-\operatorname{Lref}(\mathrm{cm})$

$(C A-F A) \times 0.8)+0.1 \times($ age -50$)-\operatorname{Lref}(\mathrm{cm})$

Lref $=$ reference distance $=$ MRI measured real traveled distance $(\mathrm{AA}-\mathrm{FA})-(\mathrm{AA}-\mathrm{CA})$

AA, ascending aorta; CA, common carotid artery; FA, common femoral artery; ${ }^{*} P<0.05$ vs. reference distance

\section{(b) Cumulative percentage of measurements differing less than 2, 5, 10 and $15 \%$ from the reference distance}

\begin{tabular}{|c|c|c|c|c|c|c|}
\hline \multirow[b]{2}{*}{$\ln \%$} & \multicolumn{2}{|c|}{ Age $<50(N=52)$} & \multicolumn{2}{|c|}{ Age $>50(N=46)$} & \multicolumn{2}{|c|}{ Total $(N=98)$} \\
\hline & $\mathbf{T}$ & TC & $\mathbf{T}$ & $\mathrm{TC}$ & $\mathbf{T}$ & TC \\
\hline$<2 \%$ difference with the reference distance & 32.7 & 50.0 & 80.4 & 69.6 & 55.1 & 59.2 \\
\hline$<5 \%$ difference with the reference distance & 46.2 & 69.2 & 93.5 & 82.6 & 68.4 & 75.5 \\
\hline$<10 \%$ difference with the reference distance & 78.8 & 96.2 & 95.7 & 95.7 & 86.7 & 95.9 \\
\hline$<15 \%$ difference with the reference distance & 98.1 & 100.0 & 100.0 & 97.8 & 99.0 & 99.0 \\
\hline
\end{tabular}

Reference distance $=M R I$ measured real traveled distance $(A A-F A)-(A A-C A) ; T=(C A-F A) \times 0.8 ; T C=[(C A-F A) \times 0.8)+(0.1 \times(a g e-50)]$.

$A A$, ascending aorta; CA, common carotid artery; FA, common femoral artery.

from longitudinal data available at that time in different populations of hypertensive patients $[17,18]$, older adults [19] and in the general population [20]. Since that time, many other studies provided either longitudinal $[2,4,21]$ or cross-sectional [7] data supporting this cut-off value. This cut-off value of $12 \mathrm{~m} / \mathrm{s}$ was based on the $100 \%$ direct common carotid artery - common femoral artery distance measurement. Adapted to the new standard distance (common carotid artery - common femoral artery $\times$ 0.8 ), it would become $9.6 \mathrm{~m} / \mathrm{s}$. We propose $10 \mathrm{~m} / \mathrm{s}$ as new standard cut-off value for cfPWV, because this is an easy figure to use in daily practice. In addition, and more important, this value is at the upper part of the second quartile in the Framingham Heart Study and represents in this general population with a mean age of 63 years about $4 \%$ risk for a first major cardiovascular event within the next 8 years [4]. One should be aware of the limitations of the use of a fixed cut-off value. The 2007 guidelines mention that 'although the relationship between aortic stiffness and events is continuous, the threshold has been suggested as a conservative estimate of significant alterations of aortic function in middle-aged hypertensive patients'. It, therefore, is not obvious that a fixed age and BP-independent cut-off value is the best risk predictor in different populations [7]. This should be further investigated.

Finally, one should keep in mind that as for BP measurement, standardization of patient conditions and repeated measurements is of utmost importance [1,22,23]. We advise to perform at least two measurements. If these differ by more than $0.5 \mathrm{~m} / \mathrm{s}$, a third measurement should be performed. The PWV value should be the median of those measurements. The median value has the advantage over the mean value that the operator does not have to calculate and that the influence of outliers is reduced or ruled out. In addition, it is also very important to take the tape measure distance in a straight line. If this is not possible as in severe obesity, an infantometer used upsidedown can provide a more reliable distance measurement. These other recommendations on user procedures are summarized below.
Other recommendations on user procedures (adapted from $[1,22,23])$ :

(1) Measurements should be performed in a quiet room with stable room temperature.

(2) Perform measurements in supine position after at least $10 \mathrm{~min}$ of rest.

(3) Measurements should preferentially be done at the right common carotid and common femoral arteries.

(4) Because of diurnal variations repeated measurements should be done at the same time of the day.

(5) No meal, caffeine or smoking is allowed within $3 \mathrm{~h}$ before measurement.

(6) Speaking and sleeping are not allowed during measurements.

(7) Data should be mean of registrations during at least one respiratory cycle (about 5-6s).

(8) Be aware of possible white coat effects.

(9) Measure distance in a straight line. If not possible with a tape measure, the upside-down use of an infantometer may be helpful.

(10) Take mean of at least two measurements; if difference between the two measurements is more than $0.5 \mathrm{~m} / \mathrm{s}$, perform a third measurement and take the median value.

(11) Situations in which measurement of cfPWV should not be performed: arrhythmia, unstable clinical situation, high-grade stenosis of carotid artery, carotid sinus syndrome.

In conclusion, this expert consensus document advises to standardize patient conditions and user procedures for the measurement of aortic stiffness using cfPWV (see Box 1). It advises to use $80 \%$ of the direct carotid-femoral (common carotid artery - common femoral artery $\times 0.8$ ) tape measure distance as new standard for daily practice, because this appears to be the most accurate body surface distance estimate, is easy to use, and is less influenced by large bellies and large breasts. In addition, it allows the use 
Box 1 Recommendations for the measurement of carotid-femoral pulse wave velocity in daily practice

- Use a dedicated and validated device to measure pulse wave travel time $(t)$ between common carotid and common femoral arteries

- Measure direct straight distance between the two measurement sites with tape measure and use $80 \%$ of this distance as pulse wave traveled distance (d)

- Calculate PWV by the formula PWV $=d / t$ (expressed as $\mathrm{m} / \mathrm{s}$ )

- Use $10 \mathrm{~m} / \mathrm{s}$ as standard cut-off value for carotid-femoral PWV in the prediction of cardiovascular events

- Take into account the other recommendations on user procedures

of the previously published normal and reference values directly without conversion [7]. Finally, this document also advises to adapt the cut-off value for cfPWV to $10 \mathrm{~m} / \mathrm{s}$.

\section{ACKNOWLEDGEMENTS}

\section{Conflicts of interest}

There are no conflicts of interest.

\section{REFERENCES}

1. Laurent S, Cockcroft J, Van Bortel L, Boutouyrie P, Giannattasio C, Hayoz D, et al. Expert consensus document on arterial stiffness: methodological issues and clinical applications. Eur Heart J 2006; 27:2588-2605.

2. Vlachopoulos C, Aznaouridis K, Stefanadis C. Prediction of cardiovascular events and all-cause mortality with arterial stiffness: a systematic review and meta-analysis. J Am College Cardiol 2010; 55:13181327.

3. Laurent S, Cockcroft J, Van Bortel L, Boutouyrie P, Giannattasio C, Hayoz D, et al., on behalf of the European Network for non ivasive investigation of large arteries. Abridged version of the expert consensus document on arterial stiffness. Artery Res 2007; 1:2-12.

4. Mitchell GF, Hwang SJ, Vasan RS, Larson MG, Pencina MJ, Hamburg NM, et al. Arterial stiffness and cardiovascular events: the Framingham Heart Study. Circulation 2010; 121:505-511.

5. Pannier B, Guerin AP, Marchais SJ, Safar ME, London GM. Stiffness of capacitive and conduit arteries: prognostic significance for end-stage renal disease patients. Hypertension 2005; 45:592-596.

6. Mancia G, De Backer G, Dominiczak A, Cifkova R, Fagard R, Germano G, et al. 2007 Guidelines for the management of arterial hypertension: The Task Force for the Management of Arterial Hypertension of the European Society of Hypertension (ESH) and of the European Society of Cardiology (ESC). J Hypertens 2007; 25:1105-1187.

7. The Reference Values for Arterial Stiffness Collaboration. Determinants of pulse wave velocity in healthy people and in the presence of cardiovascular risk factors: 'establishing normal and reference values'. Eur Heart J 2010; 31:2338-2350.

8. Sugawara J, Hayashi K, Yokoi T, Tanaka H. Carotid-femoral pulse wave velocity: impact of different arterial path length measurements. Artery Res 2010; 4:27-31.
9. Wilkinson IB, McEniery CM, Schillaci G, Boutouyrie P, Segers P, Donald A, Chowienczyk PJ, on behalf of the ARTERY Society. ARTERY Society guidelines for validation of noninvasive haemodynamic measurement devices: part 1, arterial pulse wave velocity. Artery Res 2010; 4:34-40.

10. Vermeersch SJ, Rietzschel ER, De Buyzere ML, Van Bortel LM, Gillebert TC, Verdonck PR, et al. Distance measurements for the assessment of carotid to femoral pulse wave velocity. J Hypertens 2009; 27:2377-2385.

11. Huybrechts SA, Devos DG, Vermeersch SJ, Mahieu D, Achten E, de Backer TL, et al. Carotid to femoral pulse wave velocity: a comparison of real travelled aortic path lengths determined by MRI and superficial measurements. J Hypertens 2011; 29:1577-1582.

12. Weber T, Ammer M, Rammer M, Adji A, O'Rourke MF, Wassertheurer S, et al. Noninvasive determination of carotid-femoral pulse wave velocity depends critically on assessment of travel distance: a comparison with invasive measurement. J Hypertens 2009; 27:1624-1630.

13. Filipovsky J, Mayer J, Dolejsova M, Seidlerova J. The assessment of carotid-femoral distance for aortic pulse wave velocity: should it be estimated from body height? Artery Res 2010; 4:19-23.

14. Sugawara J, Hayashi K, Yokoi T, Tanaka H. Age-associated elongation of the ascending aorta in adults. JACC 2008; 1:739-748.

15. McDonald DA. Regional pulse-wave velocity in the arterial tree. $J A p p l$ Physiol 1968; 24:73-78.

16. Van Bortel LM, Vermeersch S, De Backer T, Kips J, Huybrechts S, Segers $P$. Travel distance estimation for carotid femoral pulse wave velocity: is the gold standard a real one? J Hypertens 2011; 29:2491-2493.

17. Boutouyrie P, Tropeano AI, Asmar R, Gautier I, Benetos A, Lacolley P, Laurent S. Aortic stiffness is an independent predictor of primary coronary events in hypertensive patients: a longitudinal study. Hypertension 2002; 39:10-15.

18. Laurent S, Katsahian S, Fassot C, Tropeano AI, Gautier I, Laloux B, Boutouyrie P. Aortic stiffness is an independent predictor of fatal stroke in essential hypertension. Stroke 2003; 34:1203-1206.

19. Sutton-Tyrrell K, Najjar SS, Boudreau RM, Venkitachalam L, Kupelian V, Simonsick EM, et al. Health ABC Study. Elevated aortic pulse wave velocity, a marker of arterial stiffness, predicts cardiovascular events in well functioning older adults. Circulation 2005; 111:33843390.

20. Willum-Hansen T, Staessen JA, Torp-Pedersen C, Rasmussen S, Thijs L, Ibsen H, Jeppesen J. Prognostic value of aortic pulse wave velocity as index of arterial stiffness in the general population. Circulation 2006; 113:664-670.

21. Mattace-Raso FU, van der Cammen TJ, Hofman A, van Popele NM, Bos ML, Schalekamp MA, et al. Arterial stiffness and risk of coronary heart disease and stroke: the Rotterdam Study. Circulation 2006; 113:657663.

22. Van Bortel LM, Duprez D, Starmans-Kool MJ, Safar ME, Giannattasio C, Cockcroft J, et al. Clinical applications of arterial stiffness, Task Force III: recommendations for user procedures. Am J Hypertens 2002; $15: 445-452$.

23. Papaioannou TG, Protogerou AD, Nasothimiou EG, Tzamouranis D, Skliros N, Achimastos A, et al. Assessment of differences between repeated pulse wave velocity measurements in terms of 'bias' in the extrapolated cardiovascular risk and the classification of aortic stiffness: is a single PWV measurement enough? J Hum Hypertens 2011. doi: $10.1038 /$ jhh. 2011.76 . 